$\Rightarrow$

NON-INNOCENT LIGANDS

\title{
Guilty and charged
}

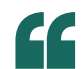

The $\mathrm{CrCl}_{2}$
(pyrazine)
material
is one of
only a few
coordination
compounds
for which
high electrical
conductivity
and strong
magnetic
interactions
coexist

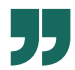

Materials that are electrically conductive and magnetic, as well as being atomically thin, are desirable for technologies in which magnetization can be finely tuned by changing either electric or spin currents. However, common 2D materials such as graphene and transition-metal dichalcogenides are non-magnetic. Conversely, metal-organic frameworks (MOFs) can be magnetic but are limited by their electrically insulating organic bridging ligands. Now, writing in Nature Chemistry, Pedersen, Clérac and co-workers describe $\mathrm{CrCl}_{2}$ (pyrazine) $)_{2}$ - a new layered coordination polymer that exhibits both conductive and magnetic-type properties.

MOFs can be prepared from an endless combination of inorganic and organic components, and redox-active examples of the latter are particularly interesting. Typical organic bridging ligands are 'innocent' in that they are redox-inactive over a normal potential window. In contrast, some ligands are 'non-innocent' and redox-active, such that they can share electrons with the metal-ion orbitals. It is precisely this redox synergy

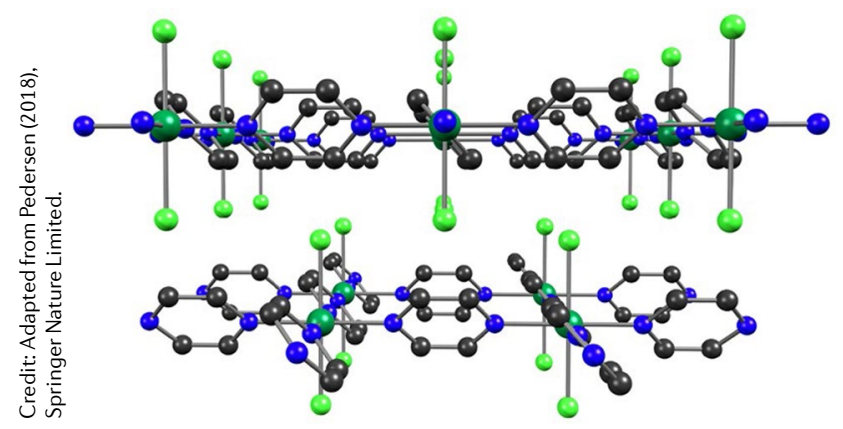

between ligands and metal ions that gives rise to the interesting properties of $\mathrm{CrCl}_{2}$ (pyrazine) ${ }_{2}$. "Our inspiration to synthesize the $\mathrm{CrCl}_{2}$ (pyrazine) coordination network was spurred by the work of Karl Wieghardt and his co-workers," explains Pedersen. "They elegantly showed that $\mathrm{Cr}^{\mathrm{II}}$ is virtually never present in a coordination environment composed of aromatic amines, as the reducing power of the former leads to an anion radical ligand and $\mathrm{Cr}^{\mathrm{III}}$. The close spatial contact between the unpaired $\mathrm{Cr}^{\mathrm{III}}$ electrons and the radical spin ensure strong magnetic interactions, which is a property we were interested in by tailoring an extended coordination network."

The group led by Pedersen and Clérac combined $\mathrm{CrCl}_{2}$ with the pyrazine ligand in one pot, with the $\mathrm{CrCl}_{2}$ (pyrazine) ${ }_{2}$ product existing as $2 \mathrm{D}$ sheets with octahedrally coordinated Cr ions. Detailed analyses were conducted to define the precise oxidation state of $\mathrm{Cr}$. The absence of the typical bond lengths of $\mathrm{Cr}^{\mathrm{II}}$ complexes in the $\mathrm{X}$-ray crystal structure of $\mathrm{CrCl}_{2}$ (pyrazine) ${ }_{2}$ and the overlap of the X-ray absorption spectra of an archetypal $\mathrm{Cr}^{\text {III }}$ complex and $\mathrm{CrCl}_{2}$ (pyrazine) ${ }_{2}$ strongly suggest the presence of $\mathrm{Cr}$ in its + III oxidation state. Furthermore, these results confirm the redox-activity of coordinated pyrazine, the presence of which can enhance the conductive and magnetic properties of a layered material. Indeed, analysis of the magnetic susceptibility as a function of temperature suggests the presence of extremely strong magnetic interactions between $\mathrm{Cr}^{\mathrm{III}}$ and the organic radical spins. Discrete Fourier transform calculations predict a strong overlap between the pyrazine $\pi$-orbitals and $\mathrm{Cr} 3 d$ orbitals. Such overlap leads to a large electron delocalization throughout the material, which results in high electrical conductivity at room temperature.

“The $\mathrm{CrCl}_{2}$ (pyrazine) ${ }_{2}$ material is one of only a few coordination compounds for which high electrical conductivity and strong magnetic interactions coexist," remarks Clérac. "Furthermore, $\mathrm{CrCl}_{2}$ (pyrazine) is a material of interest for lowdimensional magnetic and electronic applications. The structure of $\mathrm{CrCl}_{2}$ (pyrazine) is held together by strong covalent bonds within the layers and weak dispersion forces between the layers. In this way, the electronic and magnetic properties are largely confined to the 2D plane, which is itself of relevance for lowdimensional magnetism and future electronics." From a synthetic point of view, the principles investigated by Pedersen, Clérac and co-workers are by no means limited just to $\mathrm{CrCl}_{2}$ (pyrazine) ${ }_{2}$. It will then be interesting to see these synthetic principles applied to the production of different MOFs that will perhaps feature even more favourable electrical and magnetic properties.

Gabriella Graziano

ORIGINAL ARTICLE Pedersen, K. S. et al. Formation of the layered conductive magnet $\mathrm{CrCl}_{2}$ (pyrazine) ${ }_{2}$ through redox-active coordination. Nat. Chem. 10, 1056-1061 (2018) FURTHER READING Scarborough, C. C. et al. Electronic and molecular structures of the members of the electron transfer series $\left.[\mathrm{Cr} \text { (tbpy) })_{3}\right] \mathrm{n}(\mathrm{n}=3+, 2+, 1+, 0)$ : an X-ray absorption spectroscopic and density functional theoretical study. Inorg. Chem. 50, 12446-12462 (2011) 